\title{
Design of Distributed Electric Control System
}

\author{
XU Congying ${ }^{1, \text { a }}$ \\ ${ }^{1}$ Department of Automation Engineering, Zhonghuan information college Tianjin university of \\ technology, Tianjin 300380, China \\ axucongying@126.com
}

Keywords: Distributed Electric Control System; Public Building; Case Study

\begin{abstract}
As the development of electric equipment and distributed control system, the application of the distributed high-efficient system is everywhere. Being with many advantages such as environmental protection and so on, electric heating has been promoted actively in some places. As far as public buildings are concerned, distributed electric heating intelligent controlling may be the key to achieve energy conservation. In this paper, visual distributed control system was presented in electric heating of the public buildings as a case study. In this control system, PC serves as the host, and RS232 / 485 interface is regarded as converter as a serial interface conversion. Through industrial standard RS485 bus with high reliability and low cost which was the link between the host and many sets of thermostat, a one-to-many communication network was formed. With sufficient experimental analysis and implementation, we prove the robustness and effectiveness of proposed system.
\end{abstract}

\section{Introduction}

DCS is the abbreviation of the Distributed Control System. It is a multilevel computer system including control level and process monitoring levels by communication network. DCS is synthesized by Computer, Communication, CRT and Control. The basic ideas of the 4C technology are decentralized control, centralized operation, classification management and flexible configuration, easy configuration [1]. DCS control system is wildly used in the production factories and production lines, the control effect is significant. In public buildings, though some intelligent controls are used in heating system, most controlling systems are established in the one-chip computer, PLC control to realize the part of centralized control function, and hard to achieve the ideal control targets. The demand for heating is the development direction of heating in winter. Electric heating has many advantages, other heating methods don't have, such as easy to measure and control. In public buildings, energy consumption is very large, so the use electric heating will be important significance in promoting energy conservation and environmental protection. Electric heating is controllable and facilitate in measurement. So in public buildings, electric heating and intelligent control are the key to achieve intelligent energy-saving. Intelligent control of heating will be the direction of development in the future of the society.

DCS can be used in electric heating controlling system in public buildings such as schools, hospitals, hotels and so on, due to the large number of heating units, with different heating time and different heating temperature. The goal is to realize maximum heating energy-saving by careful centralized control and individual heating demand through the DCS. Control objectives are as following [2]. In many rooms to realize central control; implementation of the microcomputer program control, respectively according to the different requirements of different periods, the room; set all kinds of programs; installation of double display comprises a central control room and separate room; history, set and record keeping of the query, statistics; network can be real time query, set the program by manager; real-time data acquisition and processing to achieve the temperature, visual display real-time temperature data; temperature overrun alarm; temperature data records, including the alarm data and historical data; setting the parameters of temperature measurement, temperature parameters including temperature measurement points, the report parameter communication and parameters; temperature data report management. DCS is a crucial part of our daily life. 


\section{Design of Our Proposed System}

Structure of Control System. The public building heating DCS system is composed of a host (PC or industrial PC) as the core and multiple controllers by network [3]. High performance microcontroller and integrated sensor with high performance and high precision is adopted. Each controller on each electric heater will charge according to the host command. The RS485 bus is used to form a one to many communication networks. The controlling principle chart is as Figure 1.

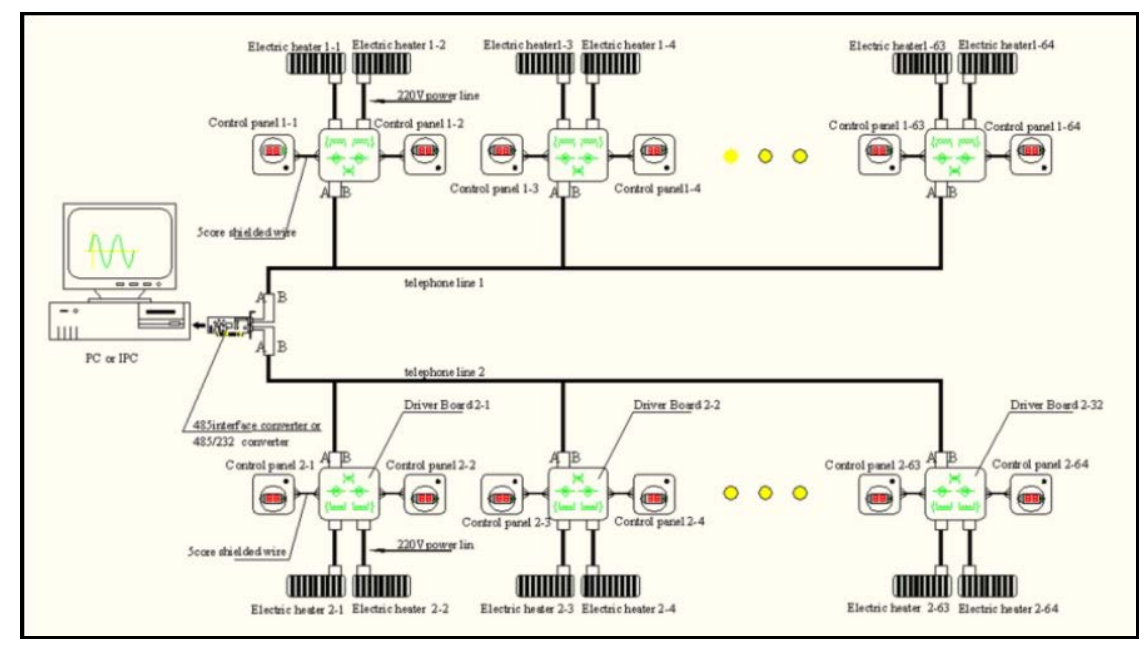

Figure. 1 The Diagram of Control System

The Main Functions of the Control Process. In DCS temperature control system, the lower computers control temperature of heaters, and receive instructions from the host using interrupt command has shown in Figure 2.

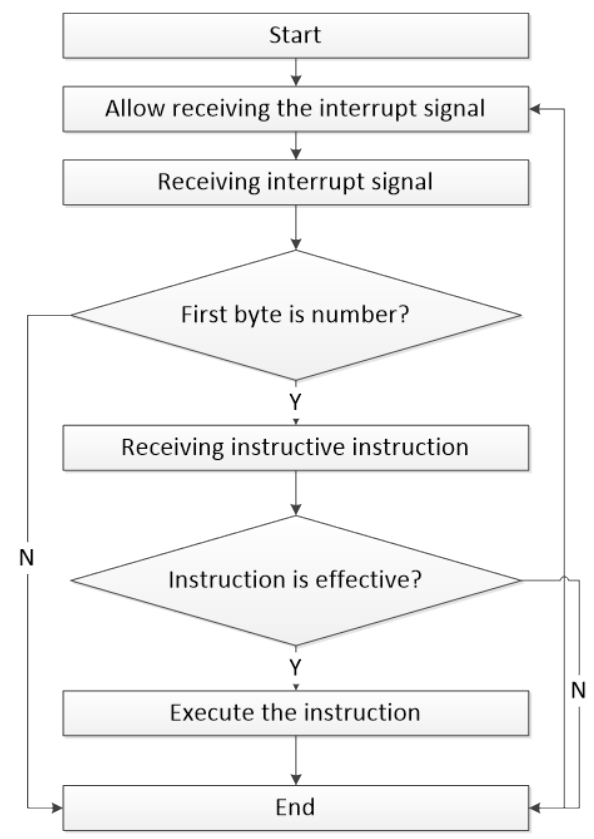

Figure. 2 The Flow Chart of Temperature Controller

Communication Protocol and Hardware. Regulation of communication protocol is as follows: a communication baud rate of $9600 \mathrm{bps}, 4 \mathrm{MHz}$ crystal oscillator [4]. The communication protocol of the temperature controller is MODBUSRTU agreement standard, including four instructions: (reading head internal digital and coil status), 0x03 (read the internal simulation and keep the memory contents), 0x05 (group within a single digital and coil status), 0x06 (set a simulation of internal single 
and are content to keep memory). DCS control system is adopted in public building heating control system to realize the operation control of the visualization. Electric heating distributed control system consists of a PC as host connecting with many temperature controllers by RS232/485 interface converters and industrial standard RS485 bus, to compose the one-to-many communication network structure and to realize communication and data processing. By Basic6.0 ActiveX control and application of Visual Basic6.0 technology [5], the effective communication distance can reach $1200 \mathrm{~m}$ under $9600 \mathrm{bps}$. The figure 3 and 4 shows the basic control panel and GUI for hardware devices.

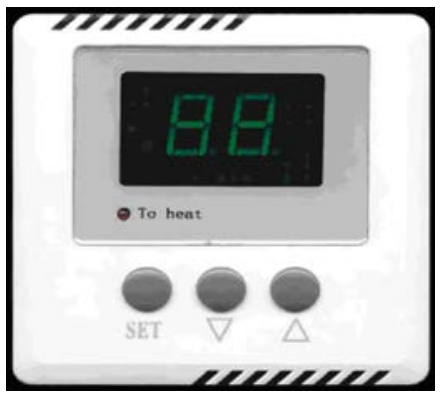

Figure. 3 The Remote Thermostat

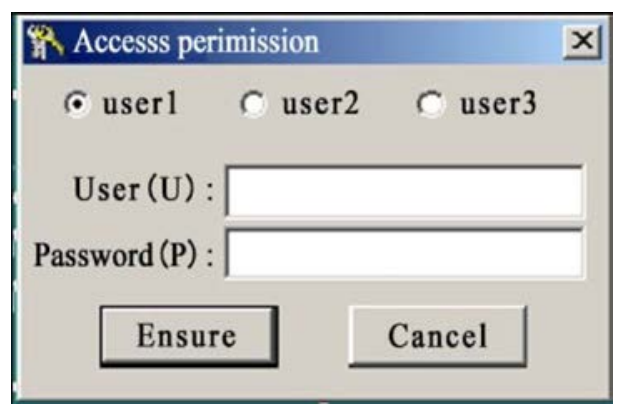

Figure. 4 The Permission GUI

\section{Experiment Analysis and Simulation}

The Installation. The installation process for our proposed system is simple. We can easily do deal with. According to the system operation process, the DCS system could be generally operated and run. In this System, three users are set to operation. Only correct user name and password are allowed to access permission of all command and control the whole system At the same time, the control permissions and fault alarm window, operation records data backup and many other functions are also designed to ensure the system working safety and efficiency just as Figure 5. DCS system is a network distributed control system, and is composed of multiple points (thermostat). So we need to set each control point of information. In this system, relevant content can be edited and deleted. Not the same as most of the current system, the installation process of our proposed system is simple and more importantly, safe and efficient.

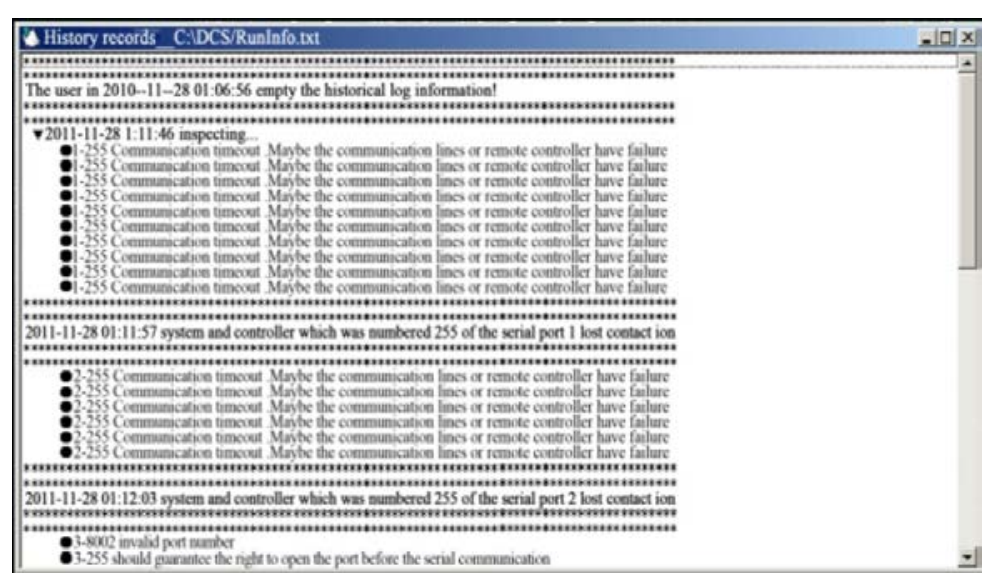

Figure. 5 The Operation Records

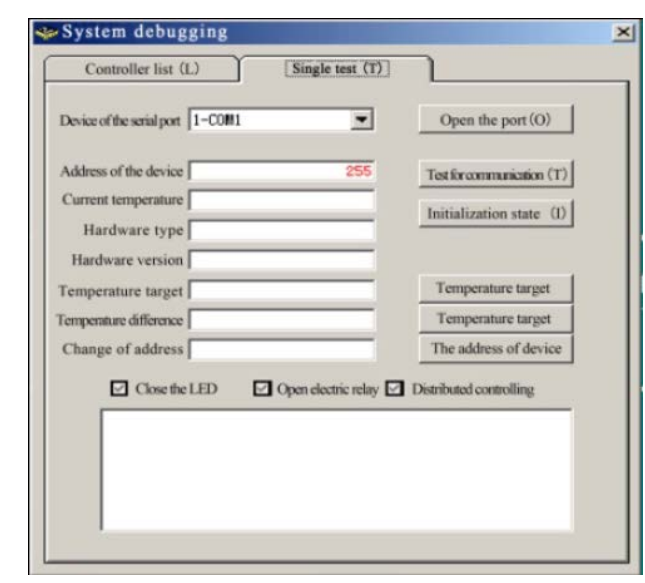

Figure. 6 The System Debugging

The System Debugging. Being numbered in advance, every room has corresponding temperature controller. When we set the system, controller can be selected according to the list. Meanwhile, a specific controller can be debugged and set address. These are showed in Figure 6. 
The Other Functions. When the system is detected and a controller loses touch, the system will timely report to the user. Then fault alarm window will be showed , and this window will keep flashing to draw attention to the operator. When the DCS system interface opens, system inspection begins immediately, then communicates and sends control commands to thermostat of each room in proper order. This control system has the function of data backup. If the hardware failure of the user computer causes the DCS system stop running which is not be repaired in a short time, through replacing a computer and installing this software ,the data will be recovered to make the DCS system perform command as normal [6].

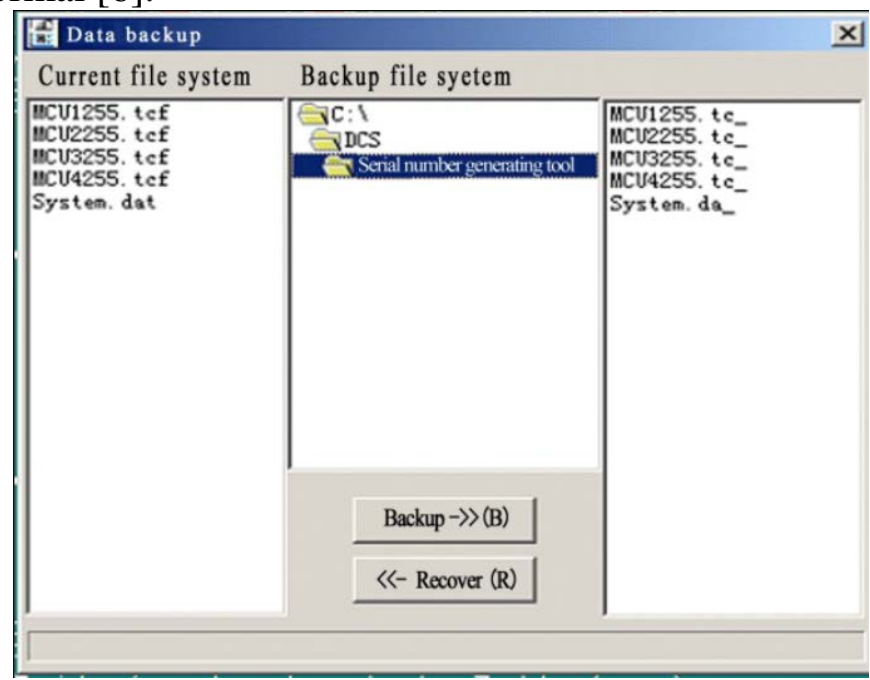

Figure. 7 The Data Backup Mechanism

\section{Summary and Conclusion}

Through this control system, the functions are realized as following: The simple, reasonable and convenient distributed control system with good performance hardware and simple software with expansibility can satisfy all sorts of control requirements to realize safety control of electric heating system in public buildings and improve its reliability and flexibility and to realize the dynamic management of each control unit, centralized control and supervision. Through the scientific control, the system guarantees warming comfortably and energy saving. Electric heating distributed control system adopts advanced system design method, is based on ActiveX controls, can realize data transmission and communication, also can be applied to drives in other different development environments. By the controls, users not only can establish control system quickly, but also expanse use scope and function according to the need. Our proposed distributed electric control system is tested for times. By comparison the electric heating using DCS control and traditional water heating, the initial investment and operation cost reduces about RMB 300 million RMB. More important points are reducing pollution and saving energy significantly.

\section{References}

[1] Luo, Yugong, et al. A Novel Hierarchical Global Chassis Control System for Distributed Electric Vehicles. No. 2014-01-0091. SAE Technical Paper, 2014.

[2] Battistelli, C., and A. J. Conejo. "Optimal management of the automatic generation control service in smart user grids including electric vehicles and distributed resources." Electric Power Systems Research 111 (2014): 22-31.

[3] Gonen, Turan. Electric power distribution engineering. CRC press, 2014.

[4] Kleinberg, Michael R., et al. "A Partitioning Method for Distributed Capacitor Control of Electric Power Distribution Systems." (2014): 1-8.

[5] Umans, Stephen D. Fitzgerald \& Kingsley's Electric Machinery. McGraw-Hill Companies, 2014. 
[6] Bai, Er-wei. "Minimizing Energy Cost in Electric Arc Furnace Steel Making by Optimal Control Designs." Journal of Energy 2014 (2014). 